Published by Al-Nahrain College of Medicine P-ISSN 1681-6579

E-ISSN 2224-4719

Email: iraqijms@colmed-alnahrain.edu.iq

http://www.colmed-alnahrain.edu.iq

http://www.iraqiims.net

Iraqi JMS 2018; Vol. 16(2)

\title{
Value of Multi-detector CT Angiography in Chronic Ischemia of Lower Limbs in Comparison with the Doppler ultrasound
}

\author{
Mohammed A. Kadhim ${ }^{1}$ FIBMS, Yaser A. Eisa ${ }^{1}$ FIBMS (CVTS), Sawsan J. Mohammed ${ }^{2}$ MBChB \\ ${ }^{1}$ Dept. of Surgery, College of Medicine, Al-Nahrain University, Baghdad, Iraq, ${ }^{2}$ Al-Imamein Al-Kadhimein Medical City, \\ Baghdad, Iraq
}

\begin{abstract}
Background

Peripheral arterial disease (PAD) is one of the most common cardiovascular diseases in developed countries and is an emerging problem in developing countries. Duplex ultrasonography (DUS) has been used as the initial imaging modality in mild symptomatic PAD. Multi-slice helical CT angiography of arteries of the thigh represents a reliable means for the detection of relevant stenoses in patients with peripheral occlusive artery disease.

Objective To assess value of multi-detector computed tomography angiography (MDCTA) and to compare it with DUS to diagnose chronic ischemia of lower limbs.

Methods

A prospective comparative study was conducted on 30 patients with chronic lower limbs ischemia of both limbs during the period from September 2015 to September 2016 at the Department of Diagnostic Radiology of Al-Imamein Al-Kadhimein Medical City, Baghdad, Iraq. DUS was done for all the patients and then MDCTA was done.

Results

Thirty patients (20 males and 10 females) with a mean age of $57.1 \pm 8.5$ (range: $33-80$ ) years were included in this study. MDCTA detects 69 lesions (41 occluded segments and 28 stenotic segments) and DUS detects 58 lesions (35 occluded segments and 43 stenotic segments). In MDCTA, 8 patients (26.7\%) had lesion in only one arterial segment, 13 patients (43.3\%) had two segment lesions, 3 patients $(10 \%)$ had three segment lesions, 4 patients $(13.3 \%)$ with four lesions and only two patients $(6.7 \%)$ had lesions in five arterial segments. Regarding the findings of the DUS one segment lesion was detected in 13 patients $(43.3 \%)$, two segment lesions in $11(36.7 \%)$, three segment lesions in $2(6.7 \%)$, four segment lesions in 3 $(10.0 \%)$ and only five segment lesions in only one patient (3.3). Furthermore, the measure of agreement between both MDCTA and DUS in the number of lesions detected revealed a good agreement between both tests, $(K a p p a=0.81)$ with a percent agreement of $(86.6 \%)$.

Conclusion Multi-detector CT angiography is a fast, accurate, safe and a minimally-invasive imaging modality which may be used in cases of PAD for diagnosis, grading and for preoperative assessment of lower limb arterial disease. Multi-detector CT Angiography, chronic ischemia of lower limbs, doppler ultrasound

Keywords

Citation

Kadhim MA, Eisa YA, Mohammed SJ. Value of multi-detector CT angiography in chronic ischemia of lower limbs in comparison with the doppler ultrasound. Iraqi JMS. 2018; 16(2): 144-151. doi: 10.22578/IJMS.16.2.5
\end{abstract}

List of abbreviations: ATA = Anterior tibial artery, $\mathrm{CLI}=$ Chronic $\operatorname{limb}$ ischemia, $\mathrm{CFA}=$ Common femoral artery, $\mathrm{CT}=$ Computed tomography, DFA = Deep femoral artery, DUS = Duplex ultrasonography, $\mathrm{MRI}=$ Magnetic resonance angiography, $\mathrm{MDCTA}=$ Multi-detector computed tomography angiography, PAD = Peripheral arterial disease, PeA = Peroneal artery, POPA $=$ Popliteal artery, PTA $=$ Posterior tibial artery, SFA = Superficial femoral artery, TCA $=$ Transcatheter angiography

\section{Introduction}

$\mathrm{P}$ eripheral arterial disease (PAD) is one of the most common cardiovascular diseases in developed countries ${ }^{(1)}$ and is an emerging issue in developing countries ${ }^{(2,3)}$. It is a manifestation of systemic atherosclerosis that commonly affects the lower extremities and is defined as any pathologic process causing obstruction to blood flow in the arteries ${ }^{(4)}$. Chronic limb ischemia (CLI) is the end result of PAD. Among aging people, and with elevating incidence of both diabetes and chronic kidney disease, chronic ischemic limb is likely to be more prevalent ${ }^{(5)}$. Existence of PAD is an extensive atherosclerosis marker ${ }^{(6)}$. The diagnosis of PAD and the subsequent treatment decisions rely on clinical exam and non-invasive imaging (7). Clinical symptoms depend most of all upon the degree of vascular stenosis/occlusion, the location of lesions in 
particular vascular segments, the degree of advancement of collateral circulation ${ }^{(5,8-10)}$. In the early stages, PAD is mostly silent. With the progression of disease, it may manifest as intermittent claudication, pain at rest ${ }^{(11)}$. Most individuals with lower extremity $P A D$ are asymptomatic and do not experience recognizable ischemic symptoms until late in the disease progression (12). Imaging is necessary for planning interventions in patients with lower extremity PAD (4).

Trans-catheter angiography (TCA) is considered as the "gold standard" for the assessment of occlusive vascular diseases of the aorta and lower extremity arteries (13). However, this method is known to be invasive and has a definite morbidity. Computed tomography (CT) scan has enormously improved during last decade ${ }^{(14,15)}$. Duplex ultrasonography (DUS) has been used as the initial imaging modality in mild symptomatic PAD (16). Color Doppler allows the rapid identification of normal and abnormal segments of vessel (17). Multi-slice helical CT angiography of arteries of the thigh represents a reliable means for the detection of relevant stenosis in patients with peripheral occlusive artery disease (18). It has the advantage of visualizing the arterial lumen and arterial wall calcifications (19). Also, it aids in good assessment of unusual lesions and identification for larger number of arterial segments, specially lesions with occlusive pathologies ${ }^{(20-23)}$. The CTA is accurate in about $87 \%$ in visualizing $>50 \%$ stenotic lesions and visualizing total obstruction in about $96 \%$, and to be $92 \%$ to $97 \%$ sensitive and of $93 \%$ to $97 \%$ regarding specificity ${ }^{(24)}$. The advantage of CTA is that it is noninvasive (unlike TCA), disadvantages are exposure to radiations, needs for potentially nephrotoxic contrast agents ${ }^{(25)}$.

This study aimed to assess the value of multi-detector computed tomography angiography (MDCTA) and to compare it with DUS in reaching the diagnosis of lower limb chronic ischemia.

\section{Methods}

This was a prospective comparative study done from September 2015 through September 2016 in Radiology Department of Al-Imamein Al-Kadhimein Medical City, Baghdad,Iraq. Thirty patients ( 20 males and 10 females) with chronic ischemic lower limbs were included regardless of their age or gender. Data were collected form patients referred from medical and cardiovascular surgery wards.

Inclusion criteria were patients with symptomatic chronic ischemic limbs.

Exclusion criteria were patient having previous interventional radiological procedures, arterial stenting or grafting, a history of significant lower limb trauma, with raised renal indices, an acute lower limb ischemia and patients with history of allergy to iodinated contrast medium.

\section{Examination protocols}

\section{Doppler ultra-sonography examinations}

Patients were examined using HD11XE (Philips medical system, Netherland). The examination was done beginning at the common femoral artery (CFA), superficial femoral artery (SFA), deep femoral artery (DFA), popliteal artery (POPA), anterior tibial artery (ATA), posterior tibial artery (PTA) and peroneal artery (PeA) were examined using a $7.5 \mathrm{MHz}$ probe. The diagnostic segment was diagnosed according to the diameter reduction less than or equal/ more than $50 \%$.

\section{Multi-detector CT angiography}

Both limbs of the patient were examined in the CT unit using (somatome definition 64 slices, Siemens medical system, Germany). CT angiography was performed following target injection of $100-120 \mathrm{ml}$ of contrast medium at a flow rate of $3-3.5 \mathrm{ml} / \mathrm{s}$ by using bolus tracking. The contrast medium used low osmolar non-ionic contrast medium (lohexol $350 \mathrm{mg} \mathrm{l} / \mathrm{ml}$ ). MDCTA was performed by using a thin section slice of $0.6 \mathrm{~mm}$.

\section{The criteria of analysis}

1) Assessment of collateral vessels; 2) opacification or non-opacification of the 
examined part; 3) Presence or absence of stenotic segments; 4) presence or absence of an occlusion with estimating its length; 5) The arterial tree was then divided into 7 segments CFA, SFA, DFA, POPA, ATA, PTA and PeA.

\section{Statistical analysis}

Patients' data were entered and analyzed using the statistical package for social sciences
(SPSS). Measure of Agreement (Kappa) (as shown in table 1) was used to assess the performance and agreement of CT angiography and duplex ultrasonography and the percent agreement was calculated, the significance level was assessed using Pearson's chi square test. Level of significance, a $P$ value $\leq 0.05$ was considered as statistically significant.

Table 1. Lower limb ischemia Benchmark scales to Kappa's value

\begin{tabular}{cc}
\hline Kappa value & Interpretation \\
\hline$<0.20$ & Poor \\
$0.21-0.40$ & Fair \\
$0.41-0.60$ & Moderate \\
$0.61-0.80$ & Good \\
$0.81-1.00$ & Very good, Almost perfect \\
\hline
\end{tabular}

\section{Results}

Thirty patients were enrolled in this study, with a mean age of $57.1 \pm 8.5$ (range: $33-80$ ) years. Males were 20 represented two thirds of the studied group, (66.7\%) and females were 10 represented the remaining (33.3\%).

According to the findings of the $\mathrm{CT}$ angiography and DUS, there were a total of $69(16.4 \%)$ and $58(13.8 \%)$ lesions detected, respectively, in both lower limbs of the 30 patients, which indicated the higher number of lesions visualized using CT angiography than DUS, however, the difference was statistically nonsignificant, $(P=0.34)$. whilst, according to the CTA 8 patients $(26.7 \%)$ had a single arterial segment lesion, 13 patients (43.3\%) had two segmental lesions, 3 patients $(10 \%)$ had three lesions, 4 patients (13.3\%) with four lesions and only two patients (6.7\%) had five artery segmental lesions. In regards to DUS findings single lesion was found in 13 patients (43.3\%), two lesions in 11 (36.7\%), three lesions in 2
(6.7\%), four lesions in $3(10.0 \%)$ and just five lesions in a single patient (3.3\%), (Table 2). Moreover, the measure of agreement between both CTA and DUS in the number of lesions found revealed a good agreement between the two tests, (Kappa $=0.81)$ with agreement percentage of (86.6\%).

Additionally, the distribution of the visualized lesions according to the type of lesion visualized and the arterial segment affected is shown in (Table 3), where the findings of CT angiography revealed that out of the 69 arterial lesions visualized to have 41 segments (68.3\%) were occluded and the remaining 28 segments (46.6\%) were found to be stenosed. The duplex ultrasonography revealed 35 (58.3\%) occluded and 23 (38.3\%) stenosed arterial segments out of the 58 total lesions detected by this test, these findings are summarized in table 3 . 
Table 2. Number and proportions of patients according to the number of affected segments detected by $\mathrm{CT}$ angiography and Doppler ultrasonography

\begin{tabular}{|c|c|c|c|c|}
\hline \multirow{2}{*}{$\begin{array}{c}\text { Number of affected } \\
\text { segments }\end{array}$} & \multicolumn{2}{|c|}{ CT Angiography } & \multicolumn{2}{|c|}{ Duplex ultrasonography } \\
\hline & No. of patients & $\%$ & No. of patients & $\%$ \\
\hline One & 8 & $26.7 \%$ & 13 & $43.3 \%$ \\
\hline Two & 13 & $43.3 \%$ & 11 & $36.7 \%$ \\
\hline Three & 3 & $10.0 \%$ & 2 & $6.7 \%$ \\
\hline Four & 4 & $13.3 \%$ & 3 & $10.0 \%$ \\
\hline Five & 2 & $6.7 \%$ & 1 & $3.3 \%$ \\
\hline Total & 30 & $100 \%$ & 30 & $100 \%$ \\
\hline \multicolumn{5}{|c|}{ Measure of Agreement for number of lesions detected } \\
\hline \multicolumn{2}{|c|}{ Kappa } & 0.81 & & \\
\hline \multirow{2}{*}{\multicolumn{2}{|c|}{$\begin{array}{l}\text { Percent agreement } \\
\qquad \text { value }<0.001\end{array}$}} & $86.6 \%$ & & \\
\hline & & nificant at & 0.05 & \\
\hline
\end{tabular}

Table 3. Distribution of lower limbs arterial lesions detected by CT angiography and doppler ultrasound according to the type of lesion and artery segment

\begin{tabular}{|c|c|c|c|c|c|c|c|c|}
\hline \multirow{3}{*}{ Artery } & \multicolumn{4}{|c|}{ Occlusion } & \multicolumn{4}{|c|}{ Stenosis } \\
\hline & \multicolumn{2}{|c|}{$\begin{array}{c}\text { CT } \\
\text { angiography }\end{array}$} & \multicolumn{2}{|c|}{$\begin{array}{l}\text { Doppler } \\
\text { Ultrasound }\end{array}$} & \multicolumn{2}{|c|}{$\begin{array}{c}\text { CT } \\
\text { angiography }\end{array}$} & \multicolumn{2}{|c|}{$\begin{array}{l}\text { Doppler } \\
\text { Ultrasound }\end{array}$} \\
\hline & No. & $\%$ & No. & $\%$ & No. & $\%$ & No. & $\%$ \\
\hline CFA & 2 & 6.7 & 2 & 6.7 & 4 & 13.3 & 3 & 10 \\
\hline DFA & 0 & 0 & 0 & 0 & 2 & 6.7 & 1 & 3.3 \\
\hline SFA & 8 & 26.7 & 6 & 20 & 5 & 16.7 & 4 & 13.3 \\
\hline POPA & 7 & 23.3 & 7 & 23.3 & 4 & 13.3 & 3 & 10 \\
\hline PTA & 8 & 26.7 & 7 & 23.3 & 4 & 13.3 & 5 & 16.7 \\
\hline ATA & 10 & 33.3 & 8 & 26.7 & 5 & 16.7 & 4 & 13.3 \\
\hline Per. A & 6 & 20 & 5 & 16.7 & 4 & 13.3 & 3 & 10 \\
\hline Total & 41 & 68.3 & 35 & 58.3 & 28 & 46.6 & 23 & 38.3 \\
\hline
\end{tabular}

The Measure of agreement between CT angiography and duplex ultrasonography regarding the type of lesions detected showed that there was a good agreement between both tests in detection of both occlusion and stenosis of the examined arterial segments, with higher percent agreement in occlusive lesions than stenosis, [(Kappa $=0.82)$, (percent agreement $=85.7 \%)$ and (Kappa $=0.75)$, (percent agreement=78.5\%), respectively, and for the detection of all lesions (Kappa $=0.79$ ) with agreement percentage of $(82.7 \%)$ as shown in table 4.

The following figures ( 1 and 2 ) show selected images of some patients presented with lower limb ischemia participated in the current study. 
Table 4. Measure of agreement between CT Angiography and Doppler ultrasonography in detection of type of arterial lesions

\begin{tabular}{cccc}
\hline & $\begin{array}{c}\text { Measure of Agreement (Kappa) } \\
\text { Kappa }\end{array}$ & Percent agreement & P value* \\
\hline In occlusion & 0.82 & $85.70 \%$ & $<0.001$ \\
In Stenosis & 0.75 & $78.50 \%$ & 0.003 \\
For all lesions & 0.79 & $82.70 \%$ & $<0.001$ \\
\hline
\end{tabular}

*P. value is significant at $\leq 0.05$
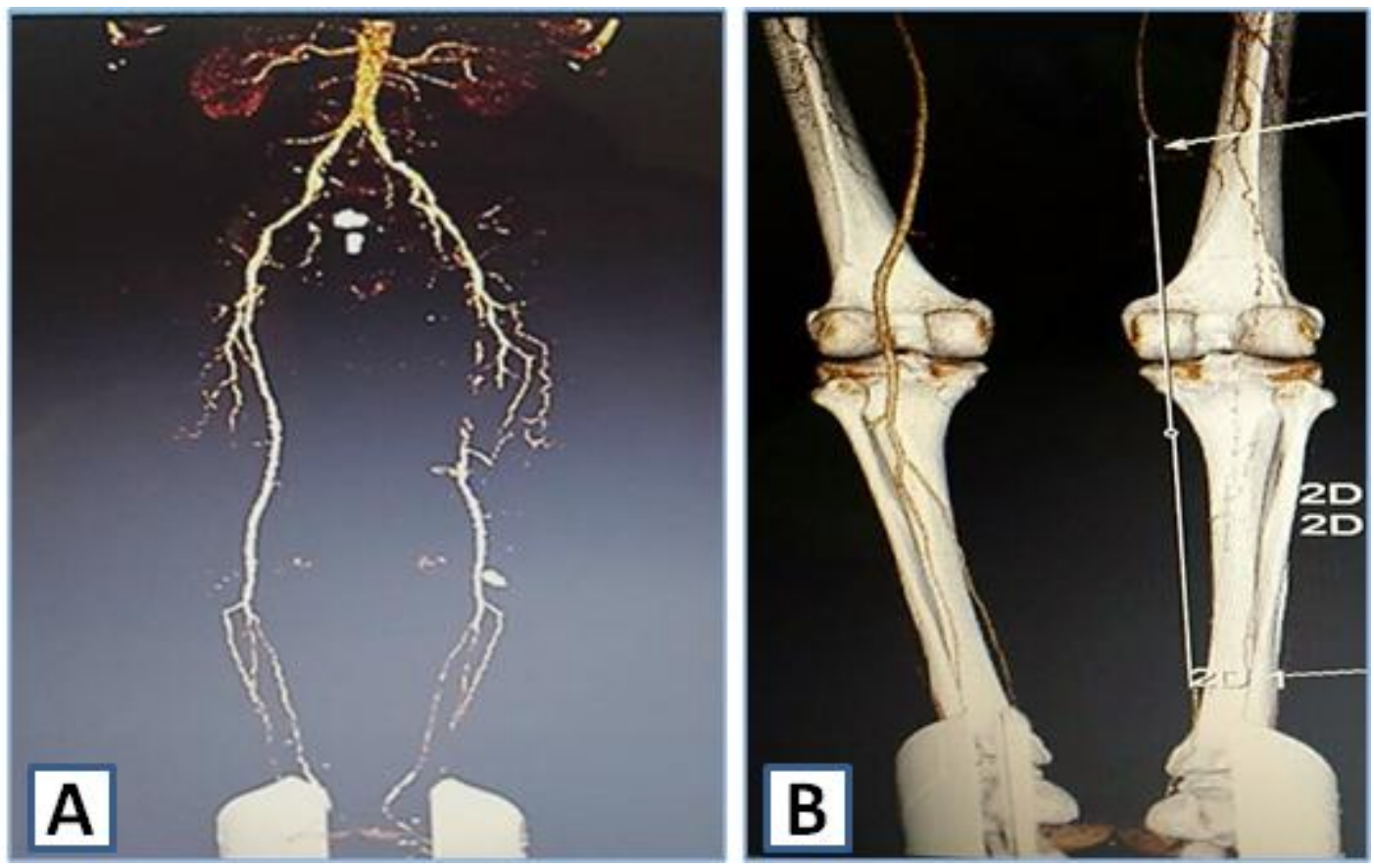

Figure 1. A: MDCTA of 60 years old male patient showing occlusion of the Left superficial femoral artery. B: MDCTA (posterior view) of 54 years old male patients presented with lower limb ischemia shows occlusion of left side distal superficial femoral, popliteal and posterior tibial arteries

\section{Discussion}

Chronic ischemic lower limb is a disease manifested via a wide range of clinical presentations, starting from being asymptomatic, through intermittent claudication, to critical limb ischemia (26). Management strategies are ruled by disease severity. Imaging is vital for planning the intervention of PAD specifically the lower limbs $(4,27)$.

Non-invasive imaging procedures, including DUS, magnetic resonance angiography (MRA), and MDCTA are available for grading lower limb arterial disease ${ }^{(6,7)}$. The DUS has been proved as a high specific and sensitive test for identification of significant hemodynamic lesions with more than $50 \%$ stenosis or occlusion (28). CT angiography continues to be attractive due to the continuous fast technical improvement, thinner slices, higher spatial resolution, short acquisition time and availability of scanning of the whole vascular tree in a limited time with a reduction in the quantity of contrast medium (29-33). CT angiography assessing the extent of PAD and provides plan and guide for vascular interventions ${ }^{(34)}$. 

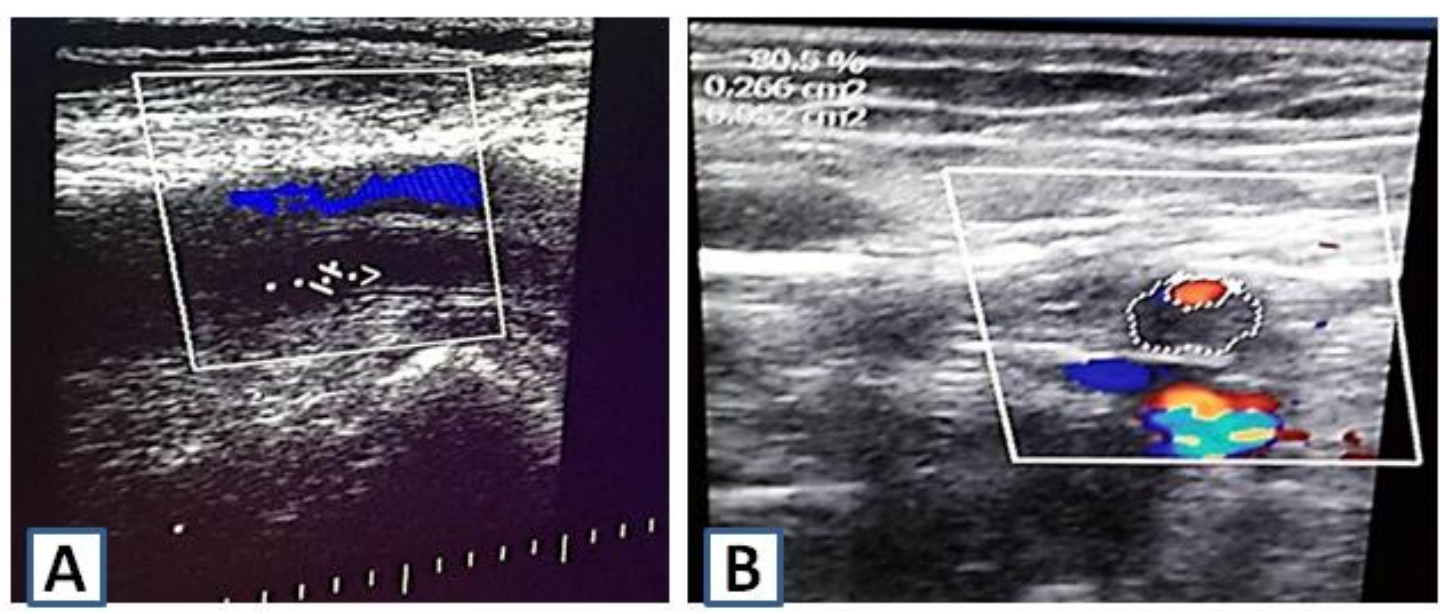

Figure 2. A: Doppler US of the Left superficial femoral artery shows no flow in color Doppler and no spectral wave in a 60 years old male patient showing occlusion of the artery. B: Color Doppler shows area of stenosis of the left femoral artery in a $\mathbf{5 4}$ years old male patient with lower limb ischemia

Various imaging techniques are used in the diagnosis of PAD. The usual is DUS and conventional angiography. The gold standard conventional angiography is responsible for complications in 1 to $2 \%$ of patients. For this reason, non-invasive techniques have been recently developed ${ }^{(32)}$.

In the present study, the MDCTA findings revealed a total of 41 occluded and 28 stenosed segments with a total lesions of 69, these findings show no great difference compared to that of DUS examination where DUS detected 58 lesions of all examined arterial segments, these lesions included 35 occlusions and 23 stenosis and according to the number of affected segments detected by MDCTA and DUS findings indicated good agreement between both tests with larger number of lesions detected on CT angiography than DUS. The cause behind this slightly lower number of lesions visualized in DUS may be due to deep anatomic position and small vascular diameter of some arterial segments which may compromise intonations seen in the peroneal artery and this show the additional value and complementary role of the CT angiography as a diagnostic tool for lower limb peripheral arterial lesions $(27,28,34)$.

The findings in regard to the good performance and good agreement rate between MDCTA and
DUS go with that reported in previous study of Pollak et al. ${ }^{(35)}$ who compared MDCTA vs DUS and found that overall, the technique for imaging vessel stenosis by using DUS is less sensitive than MDCTA and need longer time for evaluation of two lower extremities this considered the greatest limitation of DUS. Another study by Algazzar et al. ${ }^{(30)}$, revealed results differ from ours in term of no statistically difference between MDCTA and DUS, and this difference might be due to population difference, patients' inclusion criteria, and the difference in age groups of the patients in both studies.

Some studies limit the use of DUS in evaluating lower limb arterial pathologies as the procedure is totally operator dependent, it requires highly trained person, it also lacks the arterial imaging capability of MDCTA that the vascular surgeons need for preoperative planning and assessment, it can document only a small arterial segmental lesion in each image $(27,28)$.

This study concluded that MDCTA is a fast, accurate, safe and a minimally-invasive imaging modality, which may be used in cases of peripheral vascular diseases for diagnosis, grading and for preoperative assessment of lower limb arterial disease. The limiting factors that prevent the widespread usage of MDCTA 
are the limited number of multidetector row CT machines and the limited experienced stuff that can perform such a recent examination. Interpretation of the images by a radiologist with experience in vascular imaging combined with experience in multi-detector row CT imaging is mandatory.

DUS is a reliable non-invasive method of investigating the lower limb arterial system. It has an advantage over MDCTA that it provides us with hemodynamic data proximal, distal and at the site of obstruction. The limiting factor for color DUS imaging is that this examination is totally operator dependent. It requires highly trained personnel, which is not always available. It also lacks the arterial imaging capabilities of MDCTA that surgeons need for preoperative planning. It can only document a small arterial segment in each image. This leads us to the conclusion that MDCTA may replace color DUS in many cases.

\section{Acknowledgments}

Authors would like to thank the medical staff in the Radiology Department of Al-Imamein AlKadhimein Medical City for offering the opportunities of this study. Thanks a lot, to all patients who agreed to participate in this study.

\section{Authors contribution}

All the three authors were collaborated together in collecting data and writing the thesis.

\section{Conflict of interest \\ No conflict of interest is present.}

\section{Funding}

The funding of this research is all personal.

\section{References}

1. Bennett PC, Lip GY, Silverman $S$, et al. The contribution of cardiovascular risk factors to peripheral arterial disease in South Asians and Blacks: a sub-study to the Ethnic-Echocardiographic Heart of England Screening (E-ECHOES) study. QJM. 2010; 103(9): 661-9. doi: 10.1093/qjmed/hcq102.

2. Premalatha G, Shanthirani S, Deepa $R$, et al. Prevalence and Risk Factors of Peripheral Vascular Disease in a Selected South Indian Population. Diabetes Care. 2000; 23: 1295-300.
3. Weragoda J, Seneviratne R, Weerasinghe MC, et al. A cross-sectional study on peripheral arterial disease in a district of Sri Lanka: prevalence and associated factors. BMC Public Health. 2015; 15: 829. doi: 10.1186/s12889-015-2174-7.

4. Kasapis C, Gurm HS. Current approach to the diagnosis and treatment of femoral popliteal arterial disease. A systematic review. Curr Cardiol Rev. 2009; 5(4): 296-311. doi: 10.2174/157340309789317823.

5. Norgren L, Hiatt WR, Dormandy JA, et al. Intersociety consensus for the management of peripheral arterial disease (TASC II). J Vasc Surg. 2007; 45(1): S5S67. doi: http://dx.doi.org/10.1016/j.jvs.2006.12.037.

6. Selvin E, Erlinger TP. Prevalence of and risk factors for peripheral arterial disease in the United States: Results from the National Health and Nutrition Examination Survey 1999-2000. Circulation. 2004; 110(6): 738-43. doi: 10.1161/01.CIR.0000137913.26087.F0.

7. Chan $D$, Anderson ME, Dolmatch BL. Imaging evaluation of lower extremity infrainguinal disease: role of the noninvasive vascular laboratory, computed tomography angiography, and magnetic resonance angiography Tech Vasc Interv Radiol. 2010; 13(1): 11-22. doi: 10.1053/j.tvir.2009.10.003.

8. Gardner AW, Afaq Z. Management of lower extremity peripheral arterial disease. J Cardiopulm Rehabil Prev. 2008; 28(6): 349-57. doi: 10.1097/HCR.0b013e31818c3b96.

9. Walker CM, Bunch FT, Cavros NG, et al. Multidisciplinary approach to the diagnosis and management of patients with peripheral arterial disease. Clin Interv Aging. 2015; 10: 1147-53. doi: 10.2147/CIA.S79355.

10. McDermott MM, Greenland $\mathrm{P}$, Liu $K$, et al. Leg symptoms in peripheral arterial disease associated clinical characteristics and functional impairment. Journal of the American Medical Association. 2001; 286(13): 1599-606.

11. Garcia LA. Epidemiology and pathophysiology of lower extremity peripheral arterial disease. J Endovasc Ther. 2006; 13 Suppl 2: II3-9. doi: 10.1583/05-1751.1.

12. Hirsch AT, Murphy TP, Lovell MB, et al. Gaps in public knowledge of peripheral arterial disease: the first national PAD public awareness survey. Circulation. 2007; 116(18): 2086-94. doi: 10.1161/CIRCULATIONAHA.107.725101.

13. Malden ES, Picus D, Vesely TM, et al. Peripheral vascular disease: evaluation with stepping DSA and conventional screen-film angiography. Radiology. 1994; 191(1): 149-53. doi: 10.1148/radiology.191.1.8134562.

14. Rubin GD, Zarins CK. MR and spiral/helical CT imaging of lower extremity occlusive disease. Surg Clin North Am. 1995; 75(4): 607-19.

15. Reimer $P$, Landwehr $P$. Noninvasive vascular imaging of peripheral vessels. Eur Radiol. 1998; 8(6): 858-72. doi: $10.1007 /$ s003300050483. 
16. Falluji N, Mukherjee D. Critical and acute limb ischemia: an overview. Angiology. 2014; 65(2): 13746. doi: 10.1177/0003319712470966.

17. Weragoda J, Weerasinghe MC, Seneviratne R, et al. Gaps in awareness of peripheral arterial disease in Sri Lanka: a cross sectional study. BMC Public Health. 2016; 16(1): 1073. doi: 10.1186/s12889-016-3748-8.

18. Puls $R$, Knollmann $F$, Werk $M$, et al. [Multi-slice spiral CT: 3D CT angiography for evaluating therapeutically relevant stenosis in peripheral arterial occlusive disease]. Rontgenpraxis. 2001; 54(4): 141-7.

19. Laissy JP, Pernes JM. [Imaging of the lower limb arteries: when, how and why]?. J Radiol. 2004; 85(6 Pt 2): 845-50.

20. Burrill J, Dabbagh Z, Gollub F, et al. Multidetector computed tomographic angiography of the cardiovascular system. Postgrad Med J. 2007; 83(985): 698-704. doi: 10.1136/pgmj.2007.061804.

21. Romano $M$, Amato $B$, Markabaoui $K$, et al. Multidetector row computed tomography angiography of the abdominal aorta and lower limbs arteries. A new diagnostic tool in patients with peripheral arterial occlusive disease. Minerva Cardioangiol. 2004; 52(1): 9-17.

22. Mesurolle B, Qanadli S D, El Hajjam M, et al. Occlusive arterial disease of abdominal aorta and lower extremities: comparison of helical CT angiography with transcatheter angiography. Clin Imaging. 2004; 28(4): 252-60. doi: 10.1016/S08997071(03)00201-8.

23. Mishra A, Bhaktarahalli JN, Ehtuish EF. Imaging of peripheral arteries by 16-row multidetector computed tomography angiography: a feasible tool? Eur J Radiol. 2007; 61(3): 528-33. doi: 10.1016/j.ejrad.2006.10.009.

24. Antithrombotic Trialists' (ATT) Collaboration, Baigent C, Blackwell L, et al. Aspirin in the primary and secondary prevention of vascular disease: collaborative meta-analysis of individual participant data from randomised trials. Lancet. 2009; 373(9678): 1849-60. doi: 10.1016/S01406736(09)60503-1.

25. Paraskevas KI, Giannoukas AD, Kotsikoris I, et al. Contrast-induced nephropathy and the vascular patient. Angiology. 2010; 61(8): 721-3. doi: 10.1177/0003319710379110.

26. Szymczak M, Oszkinis G, Majchrzycki M. The impact of walking exercises and resistance training upon the walking distance in patients with chronic lower limb ischaemia. Biomed Res Int. 2016; 2016: 7515238. doi: 10.1155/2016/7515238.

27. Collins R, Burch J, Cranny G, et al. Duplex ultrasonography, magnetic resonance angiography, and computed tomography angiography for diagnosis and assessment of symptomatic, lower limb peripheral arterial disease: systematic review. BMJ.
2007; 334(7606): $1257 . \quad$ doi: 10.1136/bmj.39217.473275.55.

28. Androulakis AE, Giannoukas AD, Labropoulos N, et al. The impact of duplex scanning on vascular practice. Int Angiol. 1996; 15(4): 283-90.

29. Met R, Bipat $S$, Legemate $D A$, et al. Diagnostic performance of computed tomography angiography in peripheral arterial disease: a systematic review and meta-analysis. JAMA. 2009; 301(4): 415-24. doi: 10.1001/jama.301.4.415.

30. Algazzar MAA. Role of multi-detector computed tomography angiography in the evaluation of lower limb ischemia. Int J Med Imaging. 2014; 2(5): 125-30.

31. Laswed T, Rizzo E, Guntern D, et al. Assessment of occlusive arterial disease of abdominal aorta and lower extremities arteries: value of multidetector CT angiography using an adaptive acquisition method. Eur Radiol 2008; 18(2): 263-72. doi: 10.1007/s00330007-0749-0.

32. Heijenbrok-Kal MH, Kock MC, Hunink MG. Lower extremity arterial disease: multidetector CT angiography meta-analysis. Radiology. 2007;245(2): 433-9. doi: 10.1148/radiol.2451061280.

33. Catalano C, Fraioli F, Laghi A, et al. Infrarenal aortic and lower-extremity arterial disease: diagnostic performance of multi-detector row CT angiography. Radiology. 2004; 231(2): 555-63. doi: 10.1148/radiol.2312020920.

34. Duran C, Bismuth J. Advanced imaging in limb salvage. Methodist Debakey Cardiovasc J. 2012; 8(4): 28-32.

35. Pollak AW, Norton P, Kramer CM. Multimodality imaging of lower extremity peripheral arterial disease: current role and future directions. Circ Cardiovasc Imaging. 2012; 5(6): 797-807. doi: 10.1161/CIRCIMAGING.111.970814.

36. Martin ML, Tay KH, Flak B, et al. Multidetector CT angiography of the aortoiliac system and lower extremities: a prospective comparison with digital subtraction angiography. AJR Am J Roentgenol. 2003; 180(4): 1085-91. doi: 10.2214/ajr.180.4.1801085

37. Elsharawy MA, Moghazy KM. Can multi-detector computed tomographic angiography replace conventional angiography prior to lower extremity arterial reconstruction? Acta Chir Belg. 2006; 106(2): 193-8.

\section{Correspondence to dr Mohammed A. Kadhim E-mail: a_mohammed@yahoo.com mohammedal-jiboori@colmed-alnahrain.edu.iq Received Jun. $15^{\text {th }} 2017$ Accepted Dec. 25 2017}

